\title{
$\mathrm{J}$ ACS

\section{Polymer Microcapsules with Programmable Active Release}

\author{
Alireza Abbaspourrad, ${ }^{\dagger}$ Nick J. Carroll, ${ }^{\dagger}$ Shin-Hyun Kim, ${ }^{\dagger,+}$ and David A. Weitz ${ }^{* \dagger}$ \\ ${ }^{\dagger}$ School of Engineering and Applied Sciences, Department of Physics, Harvard University, Cambridge, Massachusetts 02138, United \\ States \\ ${ }^{\ddagger}$ Department of Chemical and Biomolecular Engineering, KAIST, Daejeon, South Korea
}

\section{Supporting Information}

ABSTRACT: We present a new type of microcapsule programmed with a tunable active release mechanism. The capsules are triggered by a plasticizing stimulus that induces a phase change transition of the polymeric membrane from a solid to a fluidized form; thereafter, the cargo is actively driven out of the capsule through a defect at the capsule wall with controllable release kinetics. Tuning the degree of membrane fluidity by tailoring the amount of plasticizing stimulus present allows us to obtain temporal variation of the release kinetics from a subsecond abrupt burst release to a slow sustained release of encapsulant over many minutes. Moreover, we demonstrate tuning of the collective capsule triggering

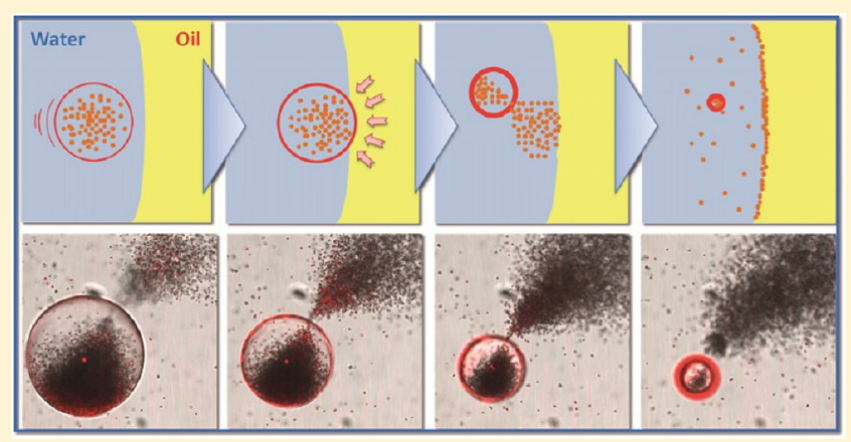
response by adjusting stimulus content, polymer molecular weight, and capsule membrane thickness. For this model system, we use a microfluidic approach to fabricate polystyrene capsules triggered by a toluene stimulus. However, this active release approach is general and is applicable to diverse polymeric capsule systems; this versatility is demonstrated by extension of our trigger-release scheme to capsules fabricated from a rubberlike block copolymer. The utility of our technique further enhances the potential of these active release capsules for practical application.

\section{INTRODUCTION}

The utility of microcapsules for efficient cargo storage and targeted release is of considerable importance in self-healing materials, ${ }^{1,2}$ nutrient preservation, ${ }^{3,4}$ fragrance release, ${ }^{5-7}$ and drug delivery. ${ }^{8-11}$ Ideal smart capsules should be composed of a membrane that effectively captures and retains actives within the core and possesses excellent specificity for triggered release by the target stimulus. ${ }^{12-17}$ The key element for fabrication of suitable structures is the design of membrane materials that can be triggered for programmable release of capsule contents; ${ }^{18-21}$ many membrane materials are tailored to undergo a chemical or physical change in response to external stimuli triggering such as $\mathrm{pH},{ }^{17-22}$ temperature, ${ }^{23}$ ultrasound, ${ }^{24,25}$ or light. ${ }^{26-30}$ The fundamental methodology of chemical response programming entails chemical reactions with the capsule membrane material initiated by a stimulus; ${ }^{18,31,32}$ for instance, disintegration of a polymeric membrane wall by chemical cleavage of cross-links. ${ }^{33}$ The precise tailoring of release profiles for chemical response capsules requires multiple functionalities incorporated into the membrane; this results in complex microcapsule architecture and synthesis.

Alternatively, physical response release occurs as a result of induced physical changes to the capsule structure. ${ }^{34,35}$ One physical response method involves vaporization of the liquid core resulting in capsule rupture due to increased internal pressure. Another technique involves an induced phase change as a result of heating the microcapsules above the melting temperature of the membrane material. ${ }^{36}$ Physical response release can also be achieved by a thermal-induced change to the porosity of a capsule membrane; for example, by the selective shrinkage of one polymeric block within a diblock copolymer membrane. ${ }^{37,38}$ However, the underlying principle of these versatile techniques is their exclusive dependence on passive release of the capsule payload. To extend the utility of microcapsules, suitable fabrication strategies are needed to provide an active release mechanism where the cargo is driven out of the capsule into the environment with controllable dispersion rates. However, fabrication of stimuli-responsive capsules that can drive out their cargo with a controllable and active release mechanism has not yet been demonstrated.

In this paper, we describe a practical approach for fabricating stimuli-responsive capsules with an active release mechanism. To trigger cargo release, we use a liquid plasticizer stimulus; the polymeric capsule membrane absorbs the stimulus, inducing a solid-to-liquid phase change within the capsule membrane. Upon membrane phase change, the intrinsic droplet interfacial instability is exploited to controllably drive out the cargo. The degree of membrane fluidity can be controlled by tailoring the amount of plasticizer present along with the molecular weight and type of membrane polymer. For our model system, we fabricate polystyrene-based capsules programmed to actively release payloads when in contact with oil. We form water-oilwater $(\mathrm{W} / \mathrm{O} / \mathrm{W})$ template double emulsion droplets to

Received: March 7, 2013

Published: April 23, 2013 

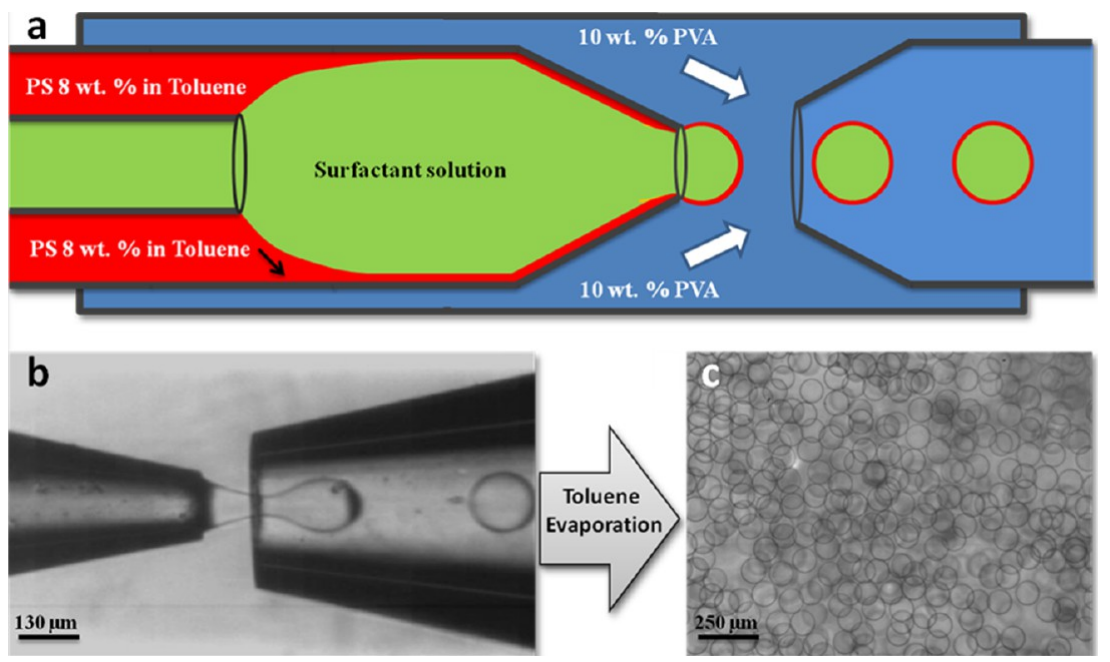

Figure 1. (a) Schematic illustration of a biphasic flow microfluidic device for fabrication of ultrathin shell double emulsion droplets. (b) Optical microscope image showing droplet formation in dripping regime. (c) Optical micrograph of resultant PS capsules with consolidated membrane following solvent evaporation treatment.

encapsulate actives within a polystyrene (PS) middle layer by exploiting a biphasic flow microfluidic technique. ${ }^{39-47}$ To obtain PS capsules, we consolidate the middle layer of the template double emulsion drops using a solvent evaporation method. The resultant PS capsule membranes are plasticized by the oil stimulus and release their payload when in contact with an oil interface. Varying the molecular weight of the membrane polymer and the thickness of the capsule membrane enables precise control of the collective capsule triggering response. We extend our approach by using block copolymers of styrene and isoprene to tailor the mechanical properties of the capsules. Our strategy provides a novel release mechanism whereby contact with a plasticizing stimulus initiates active release of capsule payload by inducing a phase change of the membrane; these capsules, with precisely tuned release profiles, have great potential in enhanced oil recovery (EOR) applications as surfactant delivery vehicles to oil-water interfaces.

\section{RESULTS AND DISCUSSION}

We use a glass capillary microfluidic device to form template double emulsion droplets with an ultrathin middle layer. ${ }^{39}$ We prepare an injection capillary by tapering a cylindrical glass capillary and subsequently treat it with $n$-octadecyltrimethoxy silane to make the inner wall hydrophobic. We insert the injection capillary into a square capillary. A tapered glass capillary is inserted coaxially into the injection capillary to facilitate simultaneous injection of two immiscible fluids. To increase the flow velocity, the flow near the injection tip is confined by inserting a cylindrical collection capillary into the square capillary from the other end; we treat this collection capillary with 2-[methoxy(polyethyleneoxy)propyl] trimethoxysilane to make the capillary wall hydrophilic. A schematic of the device is illustrated in Figure 1a.

We inject an aqueous solution of nonionic surfactant (10 wt $\%$ poly(vinyl alcohol) (PVA)) through the small tapered capillary. A solution of $8 \mathrm{wt} \%$ PS in toluene is simultaneously infused through the injection capillary. The coinjection of these two fluids results in a coaxial biphasic flow; a sheath of hydrophobic PS solution flows along the treated injection capillary wall and surrounds the innermost fluid of aqueous surfactant solution. We inject a $10 \mathrm{wt} \%$ aqueous solution of
PVA through the interstices of the square and injection capillaries to emulsify the coflowing biphasic fluids into double emulsion drops as shown in Figure 1a. Using this technique, we encapsulate an aqueous surfactant solution within an ultrathin hydrophobic middle layer of PS dissolved in toluene; a microscope image and video of droplet formation within the operating device are shown in Figure $1 \mathrm{~b}$ and Movie S1 (Supporting Information), respectively. Following droplet generation, we evaporate the toluene from the middle layer at ambient temperature to form microcapsules with a solid PS membrane. The resultant capsules with consolidated PS membranes are shown by the optical image in Figure 1c.

To distinguish capsule membrane and core structures, we label the inner fluid with a green fluorescent dye and the middle fluid with a red fluorescent dye. We use confocal microscopy to examine the core-shell structure of the capsules. The labeled microcapsule shell membrane and core are shown in the confocal images in Figure $2 \mathrm{a}$ and $\mathrm{b}$, respectively. To characterize the membrane thickness and surface morphology, we obtain scanning electron microscopy (SEM) images after drying the capsules at $45^{\circ} \mathrm{C}$. During the drying process, water from the core evaporates causing the capsule to deflate due to membrane collapse as shown in Figure 2c. Using this biphasic flow microfluidic technique allows us to form ultrathin membrane microcapsules with controllable thickness. A cross section of a capsule membrane with $200 \mathrm{~nm}$ thickness is shown in Figure 2d.

To demonstrate our concept, we use hydrocarbon oil as the plasticizing stimulus to fluidize the PS membrane and trigger the release of capsule cargo. To determine the capsule release profile as a function of membrane composition and fluidity, we add a suspension of capsules in water to hydrocarbon oil within a glass cubic cell $\left(1 \mathrm{~cm}^{3}\right)$. To form an aqueous globule $(5 \mathrm{~mm}$ diameter) in oil, the cubic cell is pretreated with $n$ octadecyltrimethoxy silane to prevent the wetting of water on the glass surface. Thereafter, we use confocal microscopy to quantify the rate of cargo release by visualizing the capsules within the cubic cell. Interestingly, in all cases, we observe a general release mechanism. First, absorption of the oil stimulus by the dispersed PS capsules in the proximity of the oil-water interface results in a phase transition of the membrane from a 


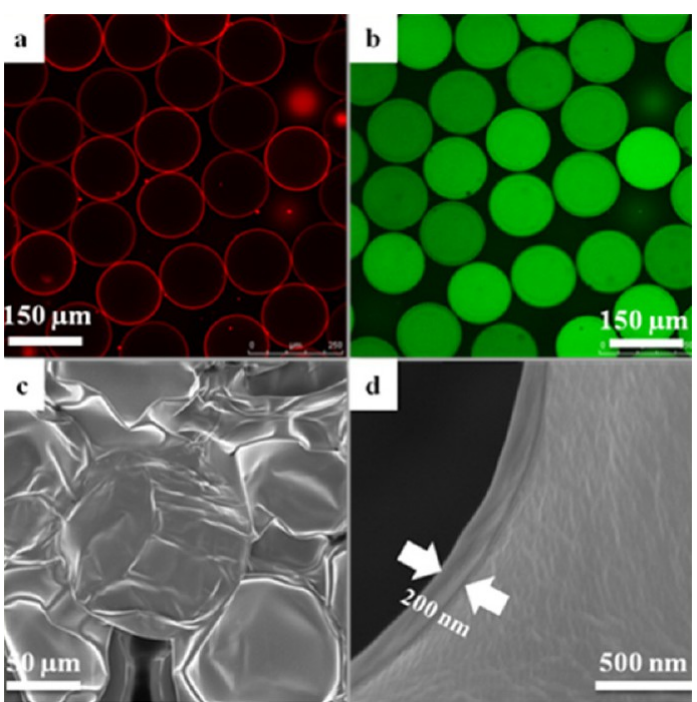

Figure 2. Confocal microscope images of (a) PS membrane labeled with Nile Red and (b) FITC-Dex labeled aqueous core. (c) SEM micrograph of obtained PS capsules and (d) SEM image of PS membrane cross section.

solid state to that of a plasticized or fluidized form. Next, the capsules contact the oil-water globule interface. Finally, a localized defect is introduced at the capsule wall, and the cargo is driven out of the capsule through this defect. This release mechanism is shown in three steps by the schematic in Figure 3. We attribute this active release behavior to the shrinkage of the plasticized membrane; the dynamic reduction of capsule size decreases the interfacial area between the plasticized PS membrane and aqueous solutions. We find that the degree of membrane fluidity directly influences the kinetics of capsule release; this provides a powerful tool to control the rate at which the cargo is driven out by tuning the fluidity of the membrane.

To investigate the effect of membrane fluidity on the kinetics of capsule release, we prepare oil solutions containing a mixture of inert linear alkanes and 10, 50, or $100 \mathrm{wt} \%$ toluene; here, toluene functions as the plasticizing stimulus. For this study, we use $400 \mathrm{kDa}$ PS to form capsule membranes with $1 \mu \mathrm{m}$ thickness and encapsulate an aqueous solution of either $10 \mathrm{wt}$ \% PVA labeled with fluorescein isothiocyanate-dextrane (FITCDex) fluorescent dye or PS tracer beads. We tune the membrane fluidity by varying the toluene stimulus content within the oil mixture and determine the corresponding release behavior. When the capsule suspension is exposed to a pure toluene stimulus, the membrane is fluidized due to absorption of toluene resulting in restoration of the capsule structure to that of a double emulsion. We observe the formation of a localized defect at the membrane wall following capsule contact with the oil-water interface; as a result, the capsule exhibits an abrupt burst of the fluidized membrane followed by a subsecond release of the encapsulated cargo. On the basis of our observations, membrane fluidization must be followed by formation of a membrane defect to initiate cargo release. During the burst release of cargo, the liquefied membrane transforms into a spherical droplet to minimize surface area as evidenced by the confocal images in Figure 4a and Movie S2 (Supporting Information). Next, we reduce the amount of toluene within the oil mixture to $50 \mathrm{wt} \%$ and examine the effect of stimulus content on the kinetics of capsule release. For clear visualization of cargo release, we encapsulate a dispersion of colloidal polystyrene in water within the core. We find that, after contact with the oil-water interface and subsequent formation of a membrane defect, the capsule shrinks while gradually driving out the payload. This gradual release process proceeds over a $75 \mathrm{~s}$ period until the payload is fully released; this is evidenced when inspecting the confocal micrograph in Figure 4b and Movie S3 (Supporting Information). This release profile is in contrast with the subsecond burst release behavior observed for the case of a pure toluene stimulus. This indicates decreasing the amount of accessible plasticizer results in a less fluidized membrane and hence a different release behavior than for the case of a pure toluene stimulus. If the toluene content is further reduced to $10 \mathrm{wt} \%$, the plasticized capsule exhibits a slow and sustained release of the encapsulant over a $12 \mathrm{~min}$ period resulting in a deflated capsule membrane as shown in Figure 4c. Furthermore, we observe no noticeable shrinkage of the nonfluidized capsule membrane during the release process as evidenced by inspection of Movie S4 (Supporting Information). Our results directly demonstrate that, by tuning the amount of available plasticizing stimulus, we can achieve orders of magnitude temporal variation of capsule release kinetics.

In the preceding experiments, we determine the release kinetics for individual capsules as a function of stimulus content. To elucidate the collective capsule triggering response, we analyze the fraction of intact capsules as a function of incubation time for three different oil stimulus mixtures. While capsules exposed to a pure toluene stimulus begin to trigger a few seconds after incubation, we observe a delay in the triggering response for capsules subjected to lower toluene content; for example, triggering does not initiate until after 20 and $30 \mathrm{~min}$ of incubation time for capsules exposed to 50 and $10 \mathrm{wt} \%$ toluene stimuli, respectively, as shown in Figure 5a. In the case of $10 \mathrm{wt} \%$ toluene stimulus, the delayed triggering provides sufficient time for the capsules to anchor and cover the oil-water interface as evidenced by the confocal Movie S5 (Supporting Information) and confocal images in Figure S1

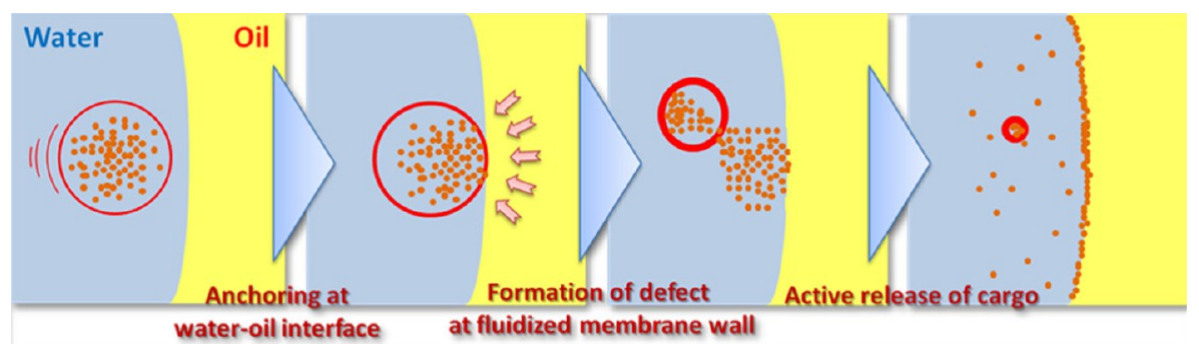

Figure 3. Schematic illustration describing capsule active release mechanism. 

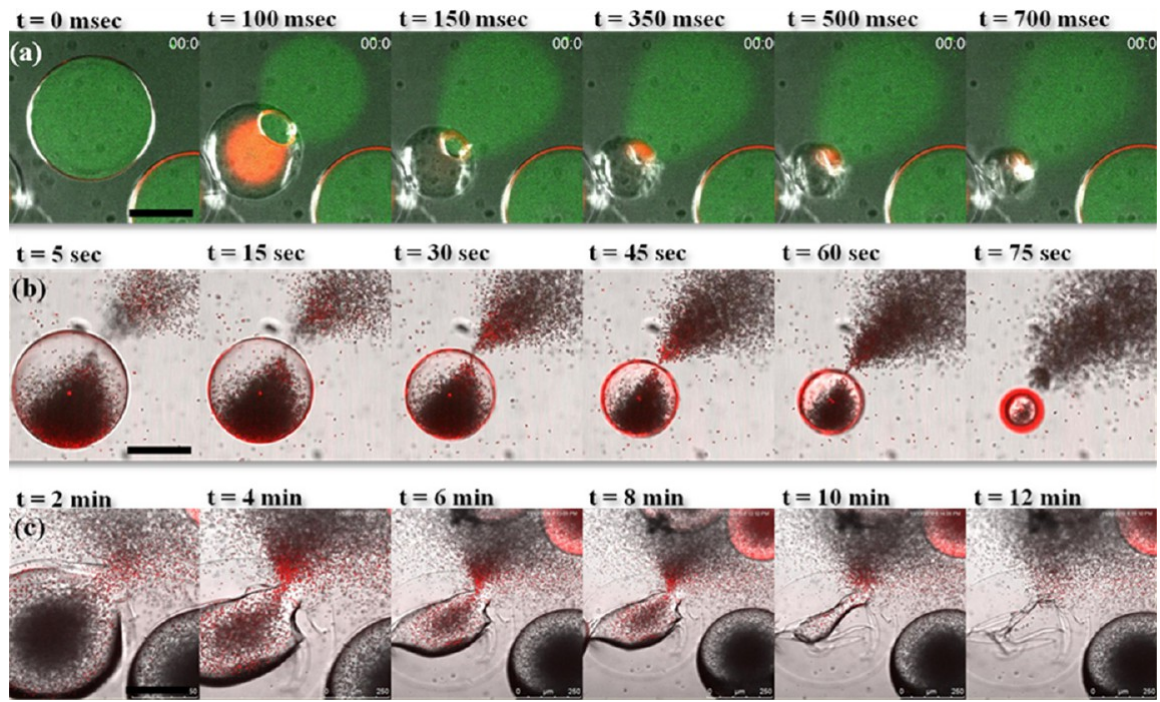

Figure 4. Time-lapse confocal microscope images showing release kinetics for capsules exposed to (a) pure toluene stimulus, (b) 50 wt $\%$ toluene stimulus, and (c) $10 \mathrm{wt} \%$ toluene stimulus.
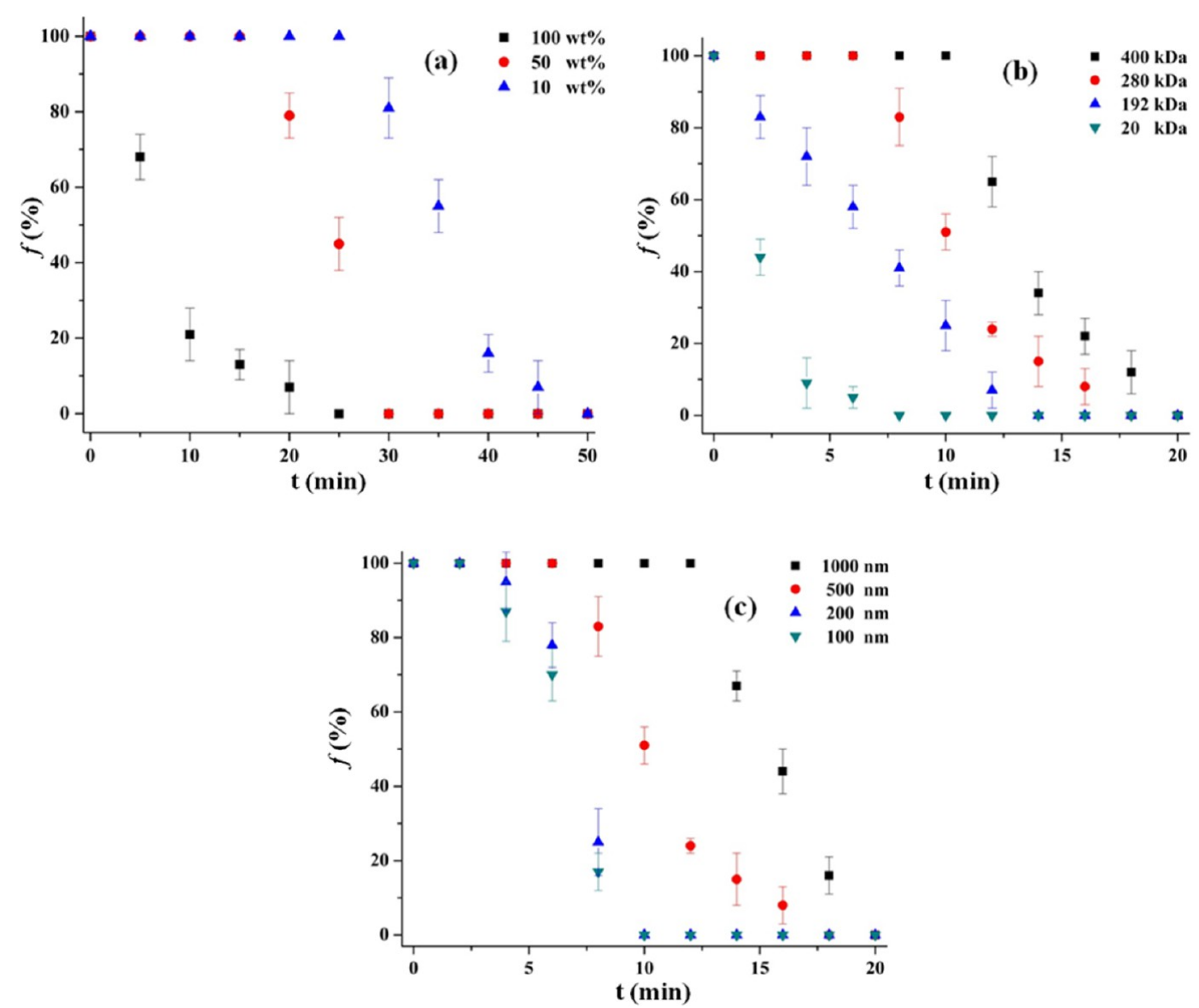

Figure 5. Fraction of intact capsules as a function of time for (a) capsules exposed to three different oil stimulus mixtures, (b) capsules fabricated using different molecular weight PS exposed to $50 \mathrm{wt} \%$ toluene stimulus, and (c) capsules with different membrane thicknesses exposed to $50 \mathrm{wt} \%$ toluene stimulus.

(Supporting Information). The strategy of controlled triggering response is not limited to tailoring of stimulus content but can be further extended by the use of membrane polymers with different molecular weight. To demonstrate this concept, we fabricate capsules with $500 \mathrm{~nm}$ membrane thickness using PS with 20,192, 280, and $400 \mathrm{kDa}$ molecular weight; we expose the resultant PS capsules to an oil mixture containing 50 wt \% toluene stimulus and determine the incubation time required for capsule triggering. Our results indicate a strong dependence of capsule triggering response on membrane polymer molecular weight; for example, capsules prepared using low molecular weight 20 or $192 \mathrm{kDa}$ PS begin to trigger seconds after stimulus 


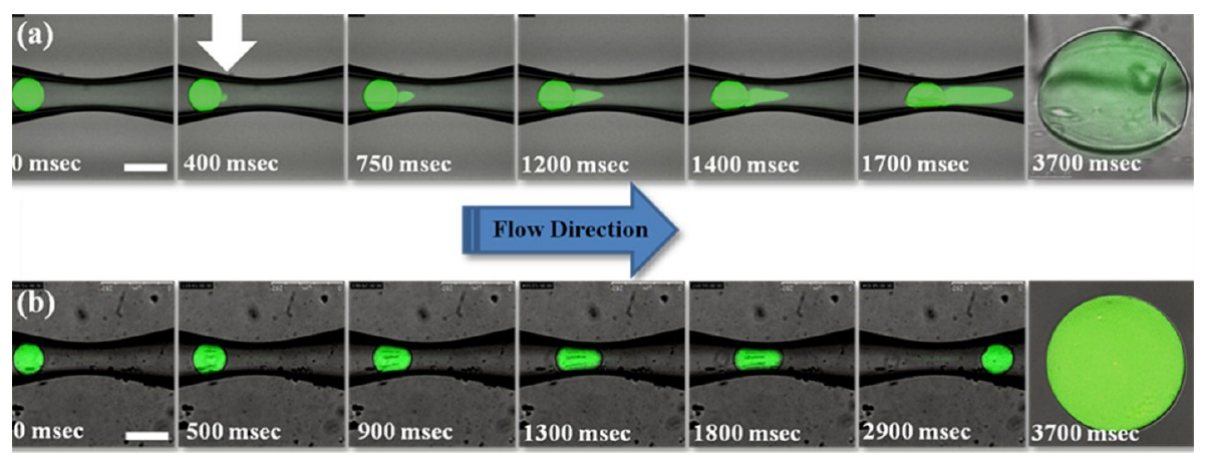

Figure 6. Confocal microscope time series images of (a) PS capsule translating through a $70 \mu \mathrm{m}$ diameter capillary constriction and (b) PS-PIP-PS capsules translating through a $70 \mu \mathrm{m}$ diameter capillary constriction. After passage through the constricting geometry the ruptured PS capsules remain buckled while the intact PS-PIP-PS capsules recover their spherical shape. Scale bars are $130 \mu \mathrm{m}$.

exposure. By contrast, triggering is significantly delayed for capsules made with large molecular weight polymer; in this case, triggering does not initiate until after 8 and $12 \mathrm{~min}$ incubation time for 280 and $400 \mathrm{kDa}$ PS, respectively, as shown in Figure $5 \mathrm{~b}$. We attribute the observed delayed triggering to increased polymeric entanglement as a result of increasing polymeric chain length. In addition to stimulus content and polymer molecular weight, the tailoring of membrane thickness also provides a simple approach to control capsule triggering response. To demonstrate this, we carry out an implementation of the preceding experiments using capsules with four different membrane thicknesses of 100, 200, 500, and $1000 \mathrm{~nm}$; we tailor the capsule membrane thickness during fabrication by adjusting the PS concentration of the droplet middle phase. For this study, we use $280 \mathrm{kDa} P S$ as the membrane material and employ an oil mixture containing $50 \mathrm{wt} \%$ toluene stimulus. Capsules with 100 and $200 \mathrm{~nm}$ membrane thickness both begin to trigger after $4 \mathrm{~min}$ of incubation time, and no significant difference in their triggering response is observed. However, by increasing the membrane thickness to $500 \mathrm{~nm}$ and $1 \mu \mathrm{m}$, we observe a considerable delay in the triggering response of 6 and 14 min incubation time, respectively, as evidenced by Figure 5c. These results demonstrate that, in addition to stimulus content and polymer molecular weight, adjusting membrane thickness provides yet another simple means to control capsule triggering response; the versatility of our technique to tailor capsule triggering response by adjusting these parameters allows customization of capsule release profiles for a variety of technological applications.

Our trigger-release strategy can easily be extended to capsules composed of diverse polymeric materials; this enables tailoring of capsule mechanical properties. In an illustrative example, we use our approach to fabricate polystyrenepolyisoprene-polystyrene (PS-PIP-PS) (styrene, 17 wt \%, Sigma-Aldrich) capsules containing an aqueous solution labeled with FITC-Dex fluorescent dye. Upon exposure to an oil stimulus consisting of $50 \mathrm{wt} \%$ toluene, the PS-PIP-PS capsules display the same gradual release behavior as observed for the PS capsules; this gradual release behavior is shown in Movie S6 (Supporting Information). Although the release behaviors of PS and PS-PIP-PS capsules are clearly similar, the capsules exhibit markedly different mechanical properties. To contrast their mechanical properties, we determine their elastic response by flowing $110 \mu \mathrm{m}$ diameter capsules through a tapered $70 \mu \mathrm{m}$ confinement, formed by pulling a $580 \mu \mathrm{m}$ diameter cylindrical glass capillary. We use a syringe pump to inject an aqueous suspension of PS capsules containing FITC-
Dex fluorescent dye into the glass capillary and visualize their passage through the constriction to probe their mechanical response. The PS capsules deform and rupture due to the imposed mechanical strain as evidenced by the release of fluorescent dye shown in the time-lapse confocal images in Figure 6a. Furthermore, the capsules remain buckled after translation through the constriction and do not recover their spherical shape as confirmed by the last frame of Figure 6a. By contrast, PS-PIP-PS capsules do not rupture while being forced through the confining geometry; the preservation of membrane integrity is substantiated by the absence of dye leakage as shown in the time-lapse confocal images of Figure $6 \mathrm{~b}$. We also observe that the PS-PIP-PS capsules ultimately recover their spherical shape as shown in the last frame of Figure $6 \mathrm{~b}$. It should be feasible to apply our trigger-release approach to polymeric capsules with distinct physical and chemical properties thus further enhancing the potential of these capsules for practical application.

\section{CONCLUSIONS}

In this work, we report fabrication of stimulus-responsive capsules with an active release mechanism. For our model system, we use PS capsules triggered for cargo release by a toluene oil mixture stimulus. The PS capsules are templated by double emulsions containing an ultrathin middle layer and are fabricated using a microfluidic and solvent evaporation approach. Cargo release is triggered by a liquid plasticizing stimulus; by absorption of the liquid plasticizing stimulus, a solid-to-liquid phase change is initiated within the capsule membrane. The capsules are triggered for release by formation of a localized defect at the membrane that is initiated by contact with the oil stimulus interface; thereafter, the cargo is actively driven out of the capsule through this defect to minimize contact area between the hydrophobic membrane and aqueous phases. We determine the dependence of capsule release kinetics on the degree of membrane fluidity by adjusting the amount of accessible plasticizer present. This allows us to achieve orders of magnitude temporal variation in the capsule release kinetics ranging from a subsecond burst release of cargo to minutes of sustained release. Furthermore, we demonstrate that, by adjusting stimulus content, polymer molecular weight, and membrane thickness, we can control capsule triggering response. This approach is not limited to a single type of membrane polymer; we demonstrate the extension of our physical release approach by fabricating capsules using a rubberlike PS-PIP-PS copolymer. Therefore, this approach should be well-suited for design of programmable release 
systems using many different types of polymeric membrane materials and plasticizing stimuli.

\section{ASSOCIATED CONTENT}

\section{S Supporting Information}

Experimental details and movies showing droplet generation, capsule trigger-release mechanisms, and capsules assembled at an oil-water interface. This material is available free of charge via the Internet at http://pubs.acs.org.

\section{AUTHOR INFORMATION}

\section{Corresponding Author}

weitz@seas.harvard.edu

\section{Notes}

The authors declare no competing financial interest.

\section{ACKNOWLEDGMENTS}

This work was supported by the Advanced Energy Consortium (http://www.beg.utexas.edu/aec/), whose member companies include BP America Inc., BG Group, Petrobras, Schlumberger, Shell, Statoil, and Total.

\section{REFERENCES}

(1) (a) Blaiszik, B. J.; Caruso, M. M.; McIlroy, D. A.; Moore, J. S.; White, S. R.; Sottos, N. R. Polymer 2009, 50, 990-997. (b) Caruso, M. M.; Schelkopf, S. R.; Jackson, A. C.; Landry, A. M.; Braun, P. V.; Moore, J. S. J. Mater. Chem. 2009, 19, 6093-6096. (c) Odom, S. A. Caruso, M. M.; Finke, A. D.; Prokup, A. M.; Ritchey, J. A.; Leonard, J. H.; Sottos, N. R.; White, S. R.; Moore, J. S. Adv. Funct. Mater. 2010, 20, 1721-1727.

(2) Brown, E. N.; White, S. R.; Sottos, N. R. J. Mater. Sci. 2004, 39, $1703-1710$.

(3) Alonso, D.; Gimeno, M.; Sepúlveda-Sánchez, J. D.; Shirai, K. Carbohydr. Res. 2010, 345, 854-859.

(4) Lee, P. S.; Yim, S. G.; Choi, Y.; Ha, T. V. A.; Ko, S. Food Chem. 2012, 134, 992-998.

(5) Zhu, G. Y.; Xiao, Z. B.; Zhou, R. J.; Yi, F. P. Adv. Mater. Res. 2012, $535,440-445$

(6) Rodrigues, C. S. N.; Martins, I. M.; Mata, V. L. G.; Barreiro, M. F.; Rodrigues, A. E. J. Text. Inst. 2012, 103, 269-282.

(7) Feczkó, T.; Kokol1, V.; Voncina, B. Macromol. Res. 2010, 18, 636-640.

(8) (a) Mertz, D.; Wu, H.; Wong, J. S.; Cui, J.; Tan, P.; Alles, R; Caruso, F. J. Mater. Chem. 2012, 22, 21434-21442. (b) He, W.; Gu, X.; Liu, S. Adv. Funct. Mater. 2012, 22, 4023-4031.

(9) (a) Shimoni, O.; Postma, A.; Yan, Y.; Scott, A. M.; Heath, J. K.; Nice, E. C.; Zelikin, A. N.; Caruso, F. ACS Nano 2012, 6, 1463-1472.

(b) Sato, K.; Yoshida, K.; Takahashi, S.; Anzai, J.-I. Adv. Drug Delivery Rev. 2011, 63, 809-821.

(10) Broaders, K. E.; Pastine, S. J.; Grandhea, S.; Frechet, J. M. J. Chem. Commun. 2011, 47, 665-667.

(11) (a) Cui, J.; Yan, Y.; Such, G. K.; Liang, K.; Ochs, C. J.; Postma, A.; Caruso, F. Biomacromolecules 2012, 13, 2225-2228. (b) Manna, U.; Patil, S. Langmuir 2009, 25, 10515-10522.

(12) (a) Lomas, H.; Johnston, A. P. R.; Such, G. K.; Zhu, Z.; Liang, K.; van Koeverden, M. P.; Alongkornchotikul, S.; Caruso, F. Small 2011, 7, 2109-2119. (b) Martino, C.; Kim, S.-H.; Horsfall, L.; Abbaspourrad, A.; Rosser, S. J.; Cooper, J.; Weitz, D. A. Angew. Chem. 2012, 51, 6416-6420.

(13) (a) Shimoni, O.; Yan, Y.; Wang, Y.; Caruso, F. ACS Nano 2013, 7, 522-530. (b) Katagiri, K.; Imai, Y.; Koumoto, K. J. Colloid Interface Sci. 2011, 361, 109-114.

(14) Zelikin, A. N.; Quinn, J. F.; Caruso, F. Biomacromolecules 2006, 7, 27-30.

(15) Kozlovskaya, V.; Kharlampieva, E.; Erelb, I.; Sukhishvili, S. A. Soft Matter 2009, 5, 4077-4087.
(16) Miguel, A. S.; Behrens, S. H. Soft Matter 2011, 7, 1948-1956.

(17) Miguel, A. S.; Scrimgeour, J.; Curtis, J. E.; Behrens, S. H. Soft Matter 2010, 6, 3163-3166.

(18) (a) Broaders, K. E.; Pastine, S. J.; Grandhe, S.; Fréchet, J. M. J. Chem. Commun. 2011, 47, 665-667. (b) Cui, L.; Cohen, J. L.; Chu, C. K.; Wich, P. R.; Kierstead, P. H.; Fréchet, J. M. J. J. Am. Chem. Soc. 2012, 134, 15840-15848.

(19) Esser-Kahn, A. P.; Odom, S. A.; Sottos, N. R; White, S. R.; Moore, J. S. Macromolecules 2011, 44, 5539-5553.

(20) Caruso, M. M.; Blaiszik, B. J.; White, S. R.; Sottos, N. R.; Moore, J. S. Adv. Funct. Mater. 2008, 18, 1898-1904.

(21) Kim, S.-H.; Shum, H. C.; Kim, J. W.; Cho, J. C.; Weitz, D. A. J. Am. Chem. Soc. 2011, 133, 15165-15171.

(22) Gil, P. R.; Nazarenus, M.; Ashraf, S.; Parak, W. J. Small 2012, 8, 943-948.

(23) Zhang, K.; Wu, W.; Guo, K.; Chen, J.; Zhang, P. Langmuir 2010, 26, 7971-7980.

(24) Pavlov, A. M.; Saez, V.; Cobley, A.; Graves, J.; Sukhorukov, G. B.; Mason, T. J. Soft Matter 2011, 7, 4341-4347.

(25) Jing, Y.; Zhu, Y.; Yang, X.; Shen, J.; Li, C. Langmuir 2011, 27, $1175-1180$.

(26) Bédard, M. F.; De Geest, B. G.; Skirtach, A. G.; Möhwald, H.; Sukhorukov, G. B. Adv. Colloid Interface Sci. 2010, 158, 2-14.

(27) Ochs, M.; Carregal-Romero, S.; Rejman, J.; Braeckmans, K.; De Smedt, S. C.; Parak, W. J. Angew. Chem. 2013, 125, 723-727.

(28) Volodkin, D.; Skirtach, A.; Mohwald, H. Polym. Int. 2012, 61, 673-679.

(29) Pastine, S. J.; Okawa, D.; Zettl, A.; Fréchet, J. M. J. J. Am. Chem. Soc. 2009, 131, 13586-13587. Kim, B.; Lee, H. S.; Kim, J.; Kim, S.-H. Chem. Commun. 2013, 49, 1865-1871.

(30) Esser-Kahn, A. P.; Sottos, N. R.; White, S. R.; Moore, J. S. J. Am. Chem. Soc. 2010, 132, 10266-10268.

(31) Price, A. D.; Zelikin, A. N.; Wang, Y.; Caruso, F. Angew. Chem. 2009, 48, 329-332.

(32) Pi, S.; Ju, X.; Wu, H.; Xie, R.; Chu, L. J. Colloid Interface Sci. 2010, 349, 512-518.

(33) Ng, S. L.; Such, G. K.; Johnston, A. P. R.; Antequera-García, G.; Caruso, F. Biomaterials 2011, 32, 6277-6284.

(34) White, S. R.; Sottos, N. R.; Geubelle, P. H.; Moore, J. S.; Kessler, M. R.; Sriram, S. R.; Brown, E. N.; Viswanathan, S. Nature 2001, 409, 794-797.

(35) Blaiszik, B. J.; Kramer, S. L. B.; Olugebefola, S. C.; Moore, J. S.; Sottos, N. R.; White, S. R. Annu. Rev. Mater. Res. 2010, 40, 179-211.

(36) Greene, L.; Phan, L. X.; Schmitt, E. E.; Mohn, J. M. Side-Chain Crystallizable Polymers for Temperature-Activated Controlled Release. In Polymeric Delivery Systems; El-Nokaly, M. A., Piatt, D. M., Charpentier, B. A., Eds.; American Chemical Society: Washington, DC, 1993; pp 244-256.

(37) Chu, L.; Park, S.; Yamaguchi, T.; Nakao, S. Langmuir 2002, 18, 1856-1864.

(38) Yang, W.; Xie, R.; Pang, X.; Ju, X.; Chu, L. J. Membr. Sci. 2008, 321, 324-330.

(39) Kim, S.-H.; Kim, J. W.; Cho, J.-C.; Weitz, D. A. Lab Chip 2011, $11,3162-3166$

(40) Tu, F.; Lee, D. Langmuir 2012, 28, 9944-9952.

(41) Lee, D.; Weitz, D. A. Adv. Mater. 2008, 20, 3498-3503.

(42) Shah, R. K.; Shum, H. C.; Rowat, A. C.; Lee, D.; Agresti, J. J.; Utada, A. S.; Chu, L.-Y.; Kim, J.-W.; Fernandez-Nieves, A.; Martinez, C. J.; Weitz, D. A. Mater. Today 2008, 11, 18-27.

(43) Utada, A. S.; Chu, L.-Y.; Fernandez-Nieves, A.; Link, D. R.; Holtze, C.; Weitz, D. A. MRS Bull. 2007, 32, 702-708.

(44) Kim, S.-H.; Kim, J.-W.; Cho, J.-C.; Weitz, D. A. Lab Chip 2011, $11,3162-3166$

(45) Ye, C.; Chen, A.; Colombo, P.; Martinez, C. J. R. Soc. Interface 2010, 7, S461-S473. Shah, R. K.; Kim, J.-W.; Agresti, J. J.; Weitz, D. A.; Chu, L.-Y. Soft Matter 2008, 4, 2303-2309.

(46) Liu, L.; Yang, J.-P.; Ju, X.-J.; Xie, R.; Liu, Y.-M.; Wang, W.; Zhang, J.-J.; Niu, C. H.; Chu, L.-Y. Soft Matter 2011, 7, 4821-4827. 
(47) Kim, S.-H.; Abbaspourrad, A.; Weitz, D. A. J. Am. Chem. Soc. 2011, 133, 5516-5525. 